[8] A. Papoulis, Probability, Random Variables, and Stochastic Processes, 3 ed. New York: McGraw-Hill.

[9] H. W. Park and C. S. Burrus, Digital Filter Design. New York: Wiley, 1987.

[10] M. E. V. Valkenburg and R. Schaumann, Design of Analog Filters. Oxford , U.K.: Oxford Univ. Press, 2001.

[11] R. Schaumann and M. E. V. Valkenburg, Design of Analog Filters. Oxford , U.K.: Oxford Univ. Press, 2001.

[12] S. Soclof, Analog Integrated Circuits. Englewood Cliffs, NJ: Prentice-Hall, 1985.

[13] W. D. Stanley and J. M. Jeffords, Electronic Communications: Principles and Systems. New York: Thompson Delmar Learning, 2006.

[14] G. Strang and T. Nguyen, Wavelets and Filter Banks. Cambridge, MA: Wellesley-Cambridge, 1996.

[15] J. F. Sturm, "Using SeDuMi 1.02, a MATLAB toolbox for optimization over symmetric cones," Optimiz. Methods Softw., pp. 625-653, 1999.

[16] H. D. Tuan, N. T. Hoang, H. Q. Ngo, T. Hoang, and B. -N. Vo, "A dual frequency-selective bounded real lemma and its application to IIR filter design," in Proc. 45th IEEE Conf. Decision Control (CDC 2006), 2006, pp. $1478-1485$

[17] P. P. Vaidyanathan, Multirate Systems and Filter Banks. Englewood Cliffs, NJ: Prentice-Hall, 1993.

\section{Improved Discrete Definition of Quadratic Time-Frequency Distributions}

\author{
John M. O' Toole, Mostefa Mesbah, and
}

Boualem Boashash, Fellow, IEEE

\begin{abstract}
Computation of a time-frequency distribution (TFD) requires a discrete version of the continuous distribution. This discrete TFD (DTFD) should be free from aliasing and conserve all the important mathematical properties of the continuous distribution. Existing DTFD definitions, however, poorly approximate this ideal. One popular definition, the generalized DTFD (GDTFD), is alias free but does not retain all the desirable properties from the continuous distribution. Another definition, the so-called alias-free GDTFD (AF-GDTFD), retains most properties yet is not always alias free. We propose a new DTFD definition, based on the GDTFD, that retains all desirable properties and is always alias free.
\end{abstract}

Index Terms-Antialiasing, discrete Fourier transforms, discrete transforms, time-frequency analysis, Wigner distributions.

\section{INTRODUCTION}

Time-frequency distributions (TFDs), which represent the joint time-frequency domain, are useful tools for extracting detailed and accurate information from nonstationary signals. Like most signal-processing methods, TFDs are usually defined for a continuous signal in the continuous domain. To compute a TFD, however, we must first define a discrete version of the distribution. Ideally, the discrete TFD should preserve all important properties from the continuous

Manuscript received October 28, 2008; accepted July 20, 2009. First published September 01, 2009; current version published January 13, 2010. The associate editor coordinating the review of this manuscript and approving it for publication was Prof. Cedric Richard.

The authors are with the University of Queensland, Centre for Clinical Research and Perinatal Research Centre, Royal Brisbane \& Women's Hospital, Herston, QLD 4029, Australia (e-mail: j.otoole@ ieee.org; j.otoole@uq.edu.au; m.mesbah@uq.edu.au; b.boashash@uq.edu.au).

This paper has supplementary material provided by the authors available to download at http://ieeexplore.ieee.org. This material consists of Matlab files to generate the discrete time-frequency distributions discussed in this paper. This material is $73 \mathrm{~KB}$ in size.

Digital Object Identifier 10.1109/TSP.2009.2031287 distribution and be free from aliasing. Yet, mapping a time-frequency distribution (TFD) from the continuous to the discrete domain may have unintended consequences - the discrete TFD may not retain all important mathematical properties from the continuous distribution, or it may be distorted by aliasing [1]-[11].

The quadratic class of TFDs $\rho(t, f)$ can be expressed in terms of two functions, the Wigner-Ville distribution (WVD) $W(t, f)$ and a kernel $\gamma(t, f)$, as

$$
\rho(t, f)=W(t, f) \underset{t \underset{f}{*}}{*} \gamma(t, f)
$$

where $*$ is the convolution operation. The kernel, which is independent of the signal, uniquely defines different members of the class. The WVD is a quadratic transformation of the real-valued signal $s(t)$

$$
W(t, f)=\int_{-\infty}^{\infty} z\left(t+\frac{\tau}{2}\right) \bar{z}\left(t-\frac{\tau}{2}\right) \mathrm{e}^{-\mathrm{j} 2 \pi \tau f} \mathrm{~d} \tau
$$

where $z(t)$ is the analytic associate of $s(t)$ [12] and $\bar{z}(t)$ is the complex conjugate of $z(t)$. Using the analytic signal avoids artefacts in the WVD between positive and negative frequency components [12].

When forming a discrete TFD (DTFD) from a discrete signal, aliasing-a distortion of the continuous TFD in the discrete domain-may occur. The DTFD may have three different types of aliasing: 1) aliasing from the wraparound artefacts caused by the circular convolution operation of the discrete WVD (DWVD) with the discrete kernel [13], 2) aliasing from the discrete analytic signal's approximation of two mutually exclusive constraints [3], [11], and 3) aliasing from the periodic overlap in the DWVD. We ignore the first and second types of aliasing because we can eliminate the first type of aliasing by replacing circular convolution with linear convolution [13] and, although we never completely eliminate the second type of aliasing, we can minimize it by using the discrete analytic signal proposed in [11]. This second type of aliasing is, typically, small compared with the third type of aliasing from the periodic overlap in the DWVD. Thus, we refer to a DTFD as alias free to mean that the DTFD is free from periodic overlap caused by the underlying DWVD.

Different DTFD definitions exist. In this paper, we consider the two popular definitions: the generalized discrete TFD (GDTFD) [5] and the alias-free GDTFD (AF-GDTFD) [6], [9]. The GDTFD is alias free but fails to satisfy all useful mathematical properties [6]. The AF-GDTFD satisfies all properties except the time- and frequency-support properties but is not always alias free [9], [10].

To address the limitations of the GDTFD and AF-GDTFD, we propose a new DTFD definition. This definition is an extension of the GDTFD because the GDTFD is a time-decimated version of the proposed definition. The proposed definition satisfies all properties and is always alias free.

\section{BACKGROUND}

In (1), we presented the quadratic TFD class as a WVD convolved with a kernel, a form that frequently appears in the literature [14]. Thus, we present the discrete quadratic class as a discrete WVD convolved with a discrete kernel. We start this section by reviewing different DWVD definitions.

\section{A. DWVD Definitions}

The DWVD uses a discrete analytic signal. This discrete signal is the analytic associate of the real-valued discrete signal $s(n T)$. We assume that $s(n T)$ is a Nyquist sampled length- $N$ signal, where $T$ is the sampling period. To simplify notation, we let $T=1$. The discrete analytic signal $z(n)$ is of the form [11]

$$
\begin{aligned}
z(n)=0, & \text { for } N \leq n \leq 2 N-1 \\
Z\left(\frac{k}{2 N}\right) \approx 0, & \text { for } N \leq k \leq 2 N-1
\end{aligned}
$$




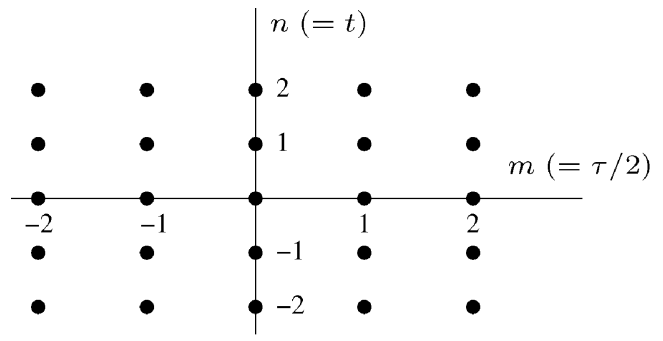

(a)

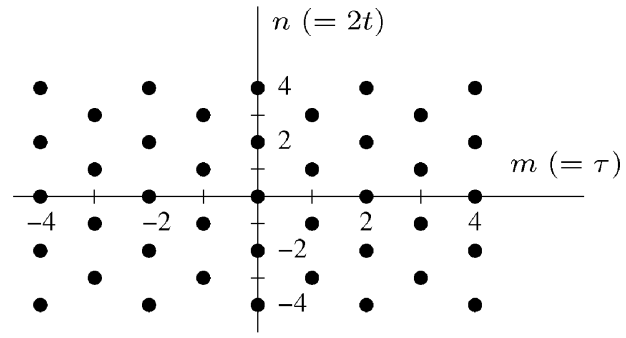

(b)

Fig. 1. Discrete time-lag grids (a) $K^{\mathrm{A}}(n, 2 m)$ and (b) $K^{\mathrm{C}}(n / 2, m)$. Dots represent the time-lag location of the sample points.

where $Z(k / 2 N)$ is the discrete Fourier transform (DFT) of $z(n)$. The approximation in (4) is necessary-a signal cannot simultaneously have a finite-time and finite-frequency bandwidth [15]. But because of this approximation, the DWVD of $z(n)$ is not completely alias free [11]. As we mentioned in the Introduction, we do not consider aliasing in the DWVD introduced by the approximation in (4) and therefore call the DWVD of $z(n)$ alias free. We refer the interested reader to a new method in [11], which improves on the standard method's approximation in (4).

Let us now examine three DWVD definitions that differ mainly in how each definition forms the discrete version of the continuous time-lag function $K(t, \tau)=z(t+\tau / 2) \bar{z}(t-\tau / 2)$ in (2). The first DWVD definition was proposed by Claasen and Mecklenbräuker [1] as

$$
W^{\mathrm{A}}\left(n, \frac{k}{2 N}\right)=\sum_{m=0}^{N-1} K^{\mathrm{A}}(n, 2 m) \mathrm{e}^{-\mathrm{j} 2 \pi m k / N}
$$

for $n, k=0,1, \ldots, N-1$, where $K^{\mathrm{A}}(n, 2 m)=z(n+m) \bar{z}(n-$ $m$ ). This discrete time-lag function $K^{\mathrm{A}}$ has a uniform discrete grid, as Fig. 1(a) shows. We call this DWVD definition DWVD-A.

The second DWVD definition $W^{\mathrm{B}}(n / 2, k / 4 N)$ was proposed by Peyrin and Prost [3] as

$$
\begin{aligned}
W^{\mathrm{B}}\left(n, \frac{k}{4 N}\right)= & \sum_{m=0}^{2 N-1} K^{\mathrm{B}}(n, 2 m) \mathrm{e}^{-\mathrm{j} \pi m k / N} \\
W^{\mathrm{B}}\left(n+\frac{1}{2}, \frac{k}{4 N}\right)= & \mathrm{e}^{-\mathrm{j} \pi k /(2 N)} \sum_{m=0}^{2 N-1} K^{\mathrm{B}}\left(n+\frac{1}{2}, 2 m+1\right) \\
& \times \mathrm{e}^{-\mathrm{j} \pi m k / N}
\end{aligned}
$$

for $n=0,1, \ldots, N-1$ and $k=0,1, \ldots, 2 N-1$, where

$$
\begin{aligned}
K^{\mathrm{B}}(n, 2 m) & =z(n+m) \bar{z}(n-m) \\
K^{\mathrm{B}}\left(n+\frac{1}{2}, 2 m+1\right) & =z(n+m+1) \bar{z}(n-m) .
\end{aligned}
$$

This time-lag function $K^{\mathrm{B}}(n / 2, m)$ exists only at the time-lag sample points $(n, 2 m)$ and $(n+1 / 2,2 m+1)$ [2]; hence the two separate expressions in (5). Fig. 1(b) illustrates this nonuniform time-lag grid. We call the DWVD in (5) the DWVD-B definition.

We proposed an alternative definition to the DWVD-B [8]. The DWVD, which we refer to as DWVD-C, is the DFT of time-lag function $K^{\mathrm{C}}$, where $K^{\mathrm{C}}$ is equal to $K^{\mathrm{B}}$ folded about $m=N$ in the lag direction. Hence, DWVD-C equals a frequency-decimated version of DWVD-B; that is, $W^{\mathrm{C}}(n / 2, k / 2 N)=W^{\mathrm{B}}(n / 2,2 k / 4 N)$. Also, DWVD-A is a decimated (in time and frequency) version of DWVD-B, as $W^{\mathrm{A}}(n, k / 2 N)=W^{\mathrm{B}}(2 n / 2,2 k / 4 N)$ [3].
The continuous and discrete distributions are closely related: DWVD-B approximates samples of the WVD [3]

$$
W^{\mathrm{B}}\left(\frac{n}{2}, \frac{k}{4 N}\right) \approx W\left(\frac{n}{2}, \frac{k}{4 N}\right)
$$

for $n, k=0,1, \ldots, 2 N-1$. This expression is not exact because of the approximation in (4). As DWVD-A and DWVD-C are also decimated versions of DWVD-B, they too approximate samples of the WVD.

\section{B. DTFD Definitions}

We now discuss two commonly used DTFD definitions based on the previously defined DWVDs. The first, known as the generalized DTFD (GDTFD), is based on the DWVD-A [5]

$$
\rho^{\mathrm{A}}\left(n, \frac{k}{2 N}\right)=W^{\mathrm{A}}\left(n, \frac{k}{2 N}\right) \underset{n}{\circledast} \underset{k}{\circledast} \gamma^{\mathrm{A}}\left(n, \frac{k}{2 N}\right)
$$

for $n, k=0,1, \ldots, N-1$, where the symbol $\circledast$ represents the circular convolution operation. The discrete time-frequency kernel $\gamma^{\mathrm{A}}$ is formed in the Doppler-lag domain $(\nu, \tau)$ by sampling $g(\nu, \tau)$ with the discrete grid $(\nu=l / N, \tau=2 m)$ to form $g^{\mathrm{A}}(l / N, 2 m)$. Mapping $g^{\mathrm{A}}(l / N, 2 m)$ to time-frequency results in the discrete kernel $\gamma^{\mathrm{A}}(n, k / 2 N)$

The GDTFD has the same periodicity as the DWVD-A [5]; that is

$$
\rho^{\mathrm{A}}\left(n, \frac{k}{2 N}\right)=\rho^{\mathrm{A}}\left(n+p N,(k+q N) \frac{1}{2 N}\right)
$$

where $p, q$ are integers. Hence, the signal information is contained within the quarter-plane time-frequency region $0 \leq t \leq N$ and $0 \leq f \leq 1 / 2$. We therefore call the GDTFD a quarter-plane distribution.

The second definition is known as the alias-free generalized DTFD [6] and is, despite its name, not always alias free [9], [10]. We present the AF-GDTFD as

$$
\rho^{\mathrm{AF}}\left(n, \frac{k}{2 N}\right)=F\left(\frac{2 n}{2}, \frac{2 k}{4 N}\right)
$$

for $n, k=0,1, \ldots, 2 N-1$, where $F$ is defined in terms of DWVD-B as

$$
F\left(\frac{n}{2}, \frac{k}{4 N}\right)=W^{\mathrm{B}}\left(\frac{n}{2}, \frac{k}{4 N}\right) \underset{n}{\circledast} \underset{k}{\circledast} \gamma^{\mathrm{AF}}\left(\frac{n}{2}, \frac{k}{4 N}\right)
$$

for $n, k=0,1, \ldots, 4 N-1$. The discrete time-frequency kernel $\gamma^{\mathrm{AF}}$ is formed in the time-lag domain by sampling the time-lag kernel $G(t, \tau)$ with the nonuniform discrete grid $(t=n, \tau=2 m)$ and $(t=n+1 / 2$, $\tau=2 m+1)$ to form $G^{\mathrm{AF}}(n / 2, m)$. This time-lag kernel has the same nonuniform sample grid as that in Fig. 1(b).

This $4 N \times 4 N$ DWVD-B in (9) is aliased [3], and therefore the AF-GDTFD may also be aliased. Thus, as we shall see in Section III-C, aliasing for the AF-GDTFD depends on the structure of the kernel. 
The AF-GDTFD, unlike the GDTFD, is periodic over the full timefrequency extent of the signal, as

$$
\rho^{\mathrm{AF}}\left(n, \frac{k}{2 N}\right)=\rho^{\mathrm{AF}}\left(n-p 2 N,(k-q 2 N) \frac{1}{2 N}\right)
$$

where $p, q$ are integers. Thus, the AF-GDTFD requires the full timefrequency region $0 \leq t \leq 2 N$ and $|f| \leq 1 / 2$ to represent the signal information. Therefore, we refer to the AF-GDTFD as a full-plane distribution.

The AF-GDTFD is related to the WVD and continuous kernel as follows:

$$
\begin{array}{r}
F\left(\frac{n}{2}, \frac{k}{4 N}\right) \approx \sum_{m=0}^{1} \sum_{l=0}^{1}(-1)^{n m+l k} W\left((n-l 2 N) \frac{1}{2},(k-m 2 N) \frac{1}{4 N}\right) \\
\times \underset{n}{\circledast} \underset{k}{\circledast} \gamma\left(\frac{n}{2}, \frac{k}{4 N}\right)
\end{array}
$$

where the AF-GDTFD is related to $F$ in (8). This relation is not exact because, as we showed in (6), the DWVD-B only approximates samples of the WVD. Thus, the AF-GDTFD does not approximate samples of the WVD convolved with a kernel but rather approximates samples of the sum of time- and frequency-shifted copies of the WVD convolved with a kernel.

Another notable DTFD definition, proposed by Nuttall [4], uses an interpolated zero-padded real-valued signal. The interpolation rate is determined by the time-frequency bandwidth of the kernel to avoid wrap around effects from circular convolution in the DTFD. Because we can express Nuttall's definition as the GDTFD of the interpolated signal, we do not consider it as a separate definition here.

\section{PROPOSED DTFD DEFINITION}

We construct the proposed DTFD definition as follows [16], [17].

1) Form the DWVD-C $W^{\mathrm{C}}(n / 2, k / 2 N)$ for $n, k=0,1, \ldots, 2 N-$ 1 .

2) Form the time-frequency kernel:

a) sample the Doppler-lag kernel $g(\nu, \tau)$ with the discrete grid $(\nu=l / N, \tau=m)$ for $l=0,1, \ldots, N-1$ and $m=$ $0,1, \ldots, 2 N-1$;

b) periodically extend this kernel $g^{\mathrm{C}}(l / N, m)$ in the Doppler $l$ direction from $N$ to $2 N$;

c) $\operatorname{map} g^{\mathrm{C}}(l / N, m)$ to the time-frequency domain to obtain the discrete kernel $\gamma^{\mathrm{C}}(n / 2, k / 2 N)$.

3) Convolve the $2 N \times 2 N$ DWVD with the $2 N \times 2 N$ kernel

$\rho^{\mathrm{C}}\left(\frac{n}{2}, \frac{k}{2 N}\right)=\left.\left[W^{\mathrm{C}}\left(\frac{n}{2}, \frac{k}{2 N}\right) \underset{n}{\circledast} \underset{k}{\circledast} \gamma^{\mathrm{C}}\left(\frac{n}{2}, \frac{k}{2 N}\right)\right]\right|_{k=0,1, \ldots, N-1}$.

The DWVD-C and kernel in (10) extend over the negative and positive frequency range. But by limiting the frequency axis to $k=0,1, \ldots, N-1$, we take only the positive frequencies. We do this because the proposed definition is a quarter-plane distribution, as

$$
\rho^{\mathrm{C}}\left(\frac{n}{2}, \frac{k}{2 N}\right)=(-1)^{q n} \rho^{\mathrm{C}}\left((n-p 2 N) \frac{1}{2},(k-q N) \frac{1}{2 N}\right)
$$

for $p, q$ integers.

To rewrite (10) as an alias-free DWVD convolved with a kernel, we express $\rho^{\mathrm{C}}(n / 2, k / 2 N T)$ in terms of $n$ and $n+1 / 2$ as follows:

$$
\begin{aligned}
\rho^{\mathrm{C}} & \left(n, \frac{k}{2 N}\right) \\
& =W^{\mathrm{C}}\left(n, \frac{k}{2 N}\right) \underset{n}{\circledast} \underset{k}{\circledast}\left[\gamma^{\mathrm{C}}\left(n, \frac{k}{2 N}\right)+\gamma^{\mathrm{C}}\left(n, \frac{k+N}{2 N}\right)\right] \\
\rho^{\mathrm{C}} & \left(n+\frac{1}{2}, \frac{k}{2 N}\right) \\
& =W^{\mathrm{C}}\left(n+\frac{1}{2}, \frac{k}{2 N}\right) \underset{n}{\circledast} \underset{k}{\circledast}\left[\gamma^{\mathrm{C}}\left(n, \frac{k}{2 N}\right)-\gamma^{\mathrm{C}}\left(n, \frac{k+N}{2 N}\right)\right] .
\end{aligned}
$$

Thus, the proposed definition is alias free. The symbol $\hat{\circledast}$ represents a modified circular convolution operation that differs from the convolution operation $\circledast$ as follows. The modified convolution operation on an arbitrary $N$-point function $a(n)$, periodic in $N$, replaces the standard periodic form of $a(n)=a(n-N)$ with the form $a(n)=-a(n-N)$.

Because, as discussed in Section II-A, the DWVD-C approximates samples of the WVD, the proposed definition approximates samples of the WVD convolved with a continuous kernel

$$
\begin{aligned}
& \rho^{\mathrm{C}}\left(n, \frac{k}{2 N}\right) \approx W\left(n, \frac{k}{2 N}\right) \\
& \circledast \underset{n}{\circledast}\left[\gamma\left(n, \frac{k}{2 N}\right)+\gamma\left(n, \frac{k+N}{2 N}\right)\right] \\
& \rho^{\mathrm{C}}\left(n+\frac{1}{2}, \frac{k}{2 N}\right) \approx W\left(n+\frac{1}{2}, \frac{k}{2 N}\right) \\
& \underset{n}{\circledast} \underset{k}{\circledast}\left[\gamma\left(n, \frac{k}{2 N}\right)-\gamma\left(n, \frac{k+N}{2 N}\right)\right] .
\end{aligned}
$$

\section{A. Properties}

Here we investigate a set of mathematical properties commonly cited in the literature [1]-[3], [6]-[9]. Each property requires a different constraint on the kernel. Some of these kernel constraints are mutually exclusive, which means that no single DTFD is capable of satisfying all properties simultaneously. The following lists the properties and the sufficient kernel constraints. Outline proofs for the properties are in the Appendix, and complete proofs are in [18].

P1) Nonnegative: The proposed DTFD is nonnegative

$$
\rho^{\mathrm{C}}\left(\frac{n}{2}, \frac{k}{2 N}\right) \geq 0
$$

if $G^{\mathrm{C}}(n / 2, m)=h(n / 2+m / 2) \bar{h}(n / 2-m / 2)$, where the time-lag kernel $G^{\mathrm{C}}$ is the inverse DFT of the time-frequency kernel $\gamma^{\mathrm{C}}$. The function $h(n)$ is zero when $n$ is not an integer.

P2) Time marginal: Summing the DTFD in the frequency direction yields the instantaneous power

$$
2 \sum_{k=0}^{N-1} \rho^{\mathrm{C}}\left(\frac{2 n}{2}, \frac{k}{2 N}\right)=|z(n)|^{2}
$$

if $G^{\mathrm{C}}(n / 2,0)=\delta(n)$.

P3) Frequency marginal: Summing the DTFD in the time direction yields the energy spectrum

$$
\sum_{n=0}^{2 N-1} \rho^{\mathrm{C}}\left(\frac{n}{2}, \frac{k}{2 N}\right)=\frac{1}{2 N}\left|Z\left(\frac{k}{2 N}\right)\right|^{2}
$$

if $\mathcal{G}^{\mathrm{C}}(0 / N, k / 2 N)=\delta(k)$, where the Doppler-frequency kernel $\mathcal{G}^{\mathrm{C}}$ is the DFT of the time-frequency kernel $\gamma^{\mathrm{C}}$.

P4) Time support: The time support of $z(n)$ is maintained in the time-frequency domain; that is, when $z(n)=0$ for $n<n_{1}$ and $n>n_{2}$, then

$$
\rho^{\mathrm{C}}\left(\frac{n}{2}, \frac{k}{2 N}\right)=0, \quad \text { for } n<2 n_{1} \text { and } n>2 n_{2}
$$

over the region $n=0,1, \ldots, 2 N-1$ and $k=0,1, \ldots, N-1$, if $G^{\mathrm{C}}(n / 2, m)=0$ for $|n|>|m|$.

P5) Frequency support: The frequency support of $Z(k / 2 N)$ is maintained in the time-frequency domain; that is, when $Z(k / 2 N)=0$ for $k<k_{1}$ and $k>k_{2}$, then

$$
\rho^{\mathrm{C}}\left(\frac{n}{2}, \frac{k}{2 N}\right)=0, \quad \text { for } k<k_{1} \text { and } k>k_{2}
$$

over the region $n=0,1, \ldots, 2 N-1$ and $k=0,1, \ldots, N-1$, if $\mathcal{G}^{\mathrm{C}}(l / N, k / 2 N)=0$ for $|k|>|l|$. 
TABLE I

PROPERTIES FOR THE DIFFERENT DTFD DEFINITIONS

\begin{tabular}{rccc}
\hline properties $^{1}$ & GDTFD & AF-GDTFD & proposed \\
\hline nonnegative & $\checkmark$ & $\checkmark$ & $\checkmark$ \\
time marginal & $\checkmark$ & $\checkmark$ & $\checkmark$ \\
frequency marginal & & $\checkmark$ & $\checkmark$ \\
time support & $\checkmark$ & & $\checkmark$ \\
frequency support & $\checkmark$ & & $\checkmark$ \\
instantaneous frequency & $\checkmark$ & $\checkmark$ & $\checkmark$ \\
group delay & & $\checkmark$ & $\checkmark$ \\
Moyal's formula & & $\checkmark$ & $\checkmark$ \\
signal recovery & & $\checkmark$ & $\checkmark$ \\
\hline
\end{tabular}

${ }^{1}$ assuming that the kernel satisfies the appropriate constraint.

P6) Instantaneous frequency: The periodic first moment [1], [19, p. 463] of the DTFD, with respect to frequency, is equal to the instantaneous frequency $f(n)$ of the signal

$\frac{1}{4 \pi}\left\{\arg \left[\sum_{k=0}^{N-1} \rho^{\mathrm{C}}\left(\frac{2 n}{2}, \frac{k}{2 N}\right) \mathrm{e}^{\mathrm{j} 2 \pi k / N}\right] \bmod 2 \pi\right\}=f(n)$

if $G^{\mathrm{C}}(n / 2,2)=a \delta(n)$, where $a$ is a positive constant. The discrete instantaneous frequency $f(n)$ is equal to the central finite difference of the phase of $z(n)$ [19, p. 463].

P7) Group delay: The periodic first moment of the DTFD, with respect to time

$-\frac{N}{2 \pi}\left\{\arg \left[\sum_{n=0}^{2 N-1} \rho^{\mathrm{C}}\left(\frac{n}{2}, \frac{k}{2 N}\right) \mathrm{e}^{-\mathrm{j} \pi n / N}\right] \bmod -2 \pi\right\}$

is equal to the group delay $\tau(k / 2 N)$ of the signal if $\mathcal{G}^{\mathrm{C}}(1 / N, k / 2 N)=a \delta(k)$, where $a$ is a positive constant. The discrete group delay $\tau(k / 2 N)$ is equal to the central finite difference of the phase of $Z(k / 2 N)$.

P8) Moyal's formula: The equality

$4 N \sum_{n=0}^{2 N-1} \sum_{k=0}^{N-1} \rho_{x}^{\mathrm{C}}\left(\frac{n}{2}, \frac{k}{2 N}\right) \rho_{y}^{\mathrm{C}}\left(\frac{n}{2}, \frac{k}{2 N}\right)=\left|\sum_{n=0}^{N-1} x(n) y(n)\right|^{2}$

know as Moyal's formula, holds if

$$
g^{\mathrm{C}}(l / N, m) \bar{g}^{\mathrm{C}}(l / N, m)=1 .
$$

P9) Signal recovery: We can recover, up to a constant phase, the time-domain signal from the DTFD

$$
2 \sum_{k=0}^{N-1} \rho^{\mathrm{C}}\left(\frac{n}{2}, \frac{k}{2 N}\right) \mathrm{e}^{\mathrm{j} \pi k n / N}=z(n) \bar{z}(0)
$$

if $G^{\mathrm{C}}(n / 2, m)=\delta(n)$ for all values of $m$.

Table I summarizes the properties for the three definitions and shows that neither the GDTFD nor the AF-GDTFD satisfies all properties [6], [9].

The DWVD should satisfy all properties except P1, nonnegativity. The DWVD associated with the GDTFD, the DWVD-A, fails to satisfy important DWVD properties such as Moyal's formula or signal recovery. The DWVD associated with the AF-GDTFD exists only when the signal length is odd [9]; but as $z(n)$ is length $2 N$, the AF-GDTFD does not contain a DWVD definition. The authors proposed a smoothed DWVD for the AF-GDTFD for even-length signals that, similar to DWVD-A, does not satisfy Moyal's formula or the signal recovery

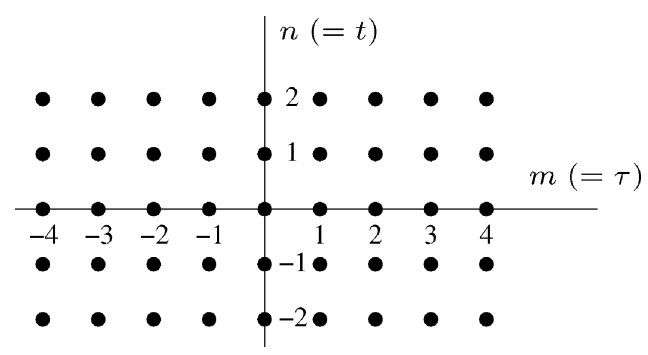

Fig. 2. Discrete time-lag grid for $G^{\mathrm{C}}(n / 2, m)$. Dots show the time-lag location of sample points. Sample points at $n / 2$ noninteger values are zero; that is, $G^{\mathrm{C}}(n+1 / 2, m)=0$.

property. In contrast, the DWVD associated with the proposed definition, the DWVD-C, satisfies properties P2-P9 [8].

\section{B. Relation to the GDTFD and AF-GDTFD}

The GDTFD is a specific case of the proposed definition, as we can obtain the GDTFD from the proposed definition by simply decimating the latter in time- that is

$$
\rho^{\mathrm{C}}\left(\frac{2 n}{2}, \frac{k}{2 N}\right)=\rho^{\mathrm{A}}\left(n, \frac{k}{2 N}\right) .
$$

We obtain this expression by substituting $\gamma^{\mathrm{A}}(n, k / 2 N)=$ $\gamma^{\mathrm{C}}(n, k / 2 N)+\gamma^{\mathrm{C}}(n,(k+N) / 2 N)$ into (12) and comparing this result with the definition of the GDTFD in (7). The kernel $\gamma^{\mathrm{A}}(n, k / 2 N)$ equals a folded (in frequency) version of $\gamma^{\mathrm{C}}(n, k / 2 N)$ because the kernel $g^{\mathrm{A}}(l / N, 2 m)$ is a decimated (in lag) version of the kernel $g^{\mathrm{C}}(l / N, m)$. A consequence of the decimation process in (13) is that the $N \times N$ GDTFD requires only half of the computational load to compute compared with the load for the $2 N \times N$ proposed definition [17].

The proposed definition is considerably different, however, from the AF-GDTFD. First, the extent of the Doppler-lag kernels differ: the AF-GDTFD's kernel extends over the region $|\nu| \leq 1$ and $|\tau| \leq 2 N$, whereas the proposed definition's kernel extends over the smaller region $|\nu| \leq 1 / 2$ and $|\tau| \leq N$. Second, the time-lag kernels have different discrete grids: the AF-GDTFD's kernel has a nonuniform discrete grid [9], same as the grid in Fig. 1(b), whereas the proposed DTFD's kernel has a uniform discrete grid, illustrated in Fig. 2. Third, the AF-GDTFD is a full-plane distribution - periodic over the time-frequency region $0 \leq t \leq 2 N$ and $|f| \leq 1 / 2$-whereas the proposed DTFD is a quarter-plane distribution-periodic over the smaller region $0 \leq t \leq N$ and $0 \leq f \leq 1 / 2$. In addition, the $2 N \times 2 N$ AF-GDTFD requires twice the computational load to compute compared with the load for the $2 N \times N$ proposed definition.

\section{Numerical Example}

We present an example to show that the AF-GDTFD is not always alias free, whereas the proposed definition is always alias free. We do not show the GDTFD here because it is simply a time-decimated version of the proposed definition.

The example uses a linear frequency modulated (LFM) test signal $s_{1}(n)=\cos \left(2 \pi\left(0.1 n+0.4 n^{2} / 128\right)\right)$ for $n=0,1, \ldots, N-1$, where $N=64$. We transformed $s_{1}(n)$ into the $2 N$-point analytic signal $z_{1}(n)$ using the method in [11]. Each definition uses three different DTFDs - a lag-independent kernel DTFD, a Doppler-independent kernel DTFD, and a separable kernel DTFD [14]. Fig. 3 shows the DTFD plots.

The signal energy for the AF-GDTFD, which is a full-plane distribution, should be contained within the quadrant $0 \leq t \leq N$ and 
AF-GDTFD

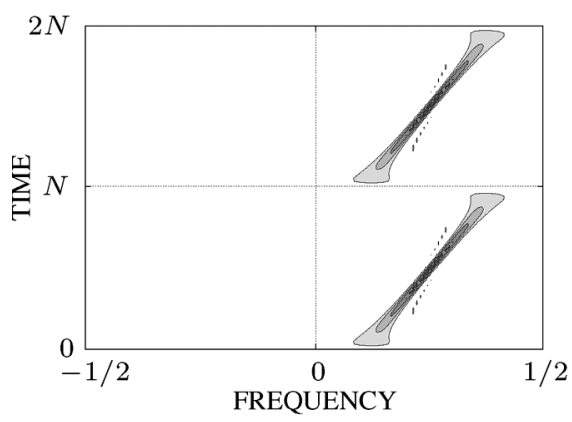

PROPOSED

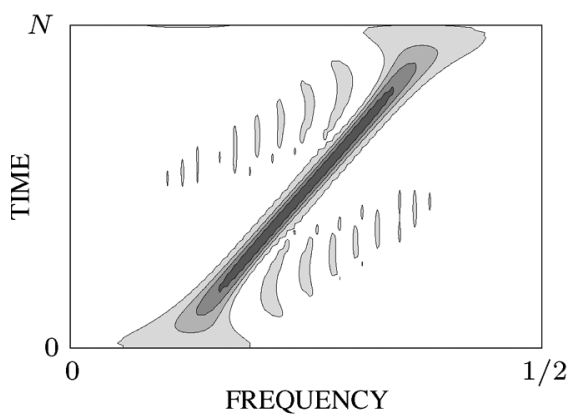

AF-GDTFD

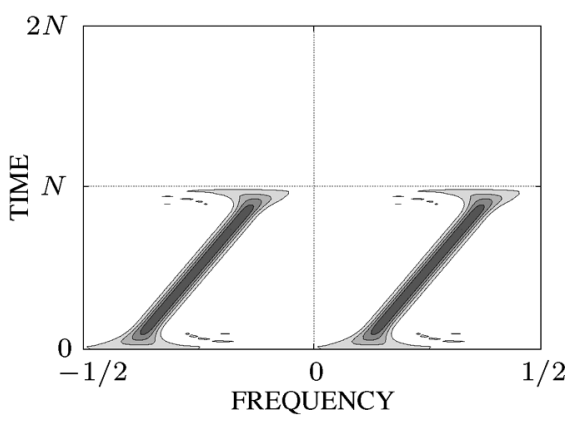

(a)

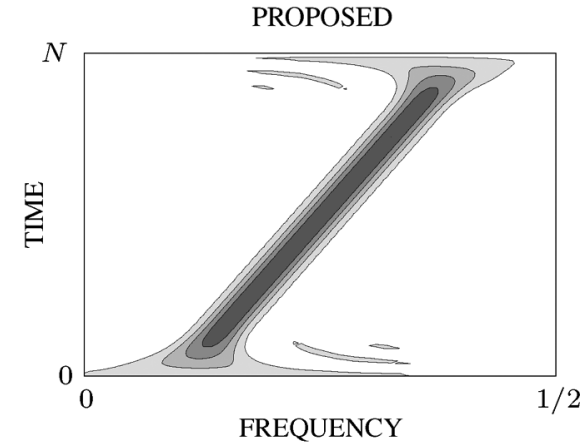

AF-GDTFD

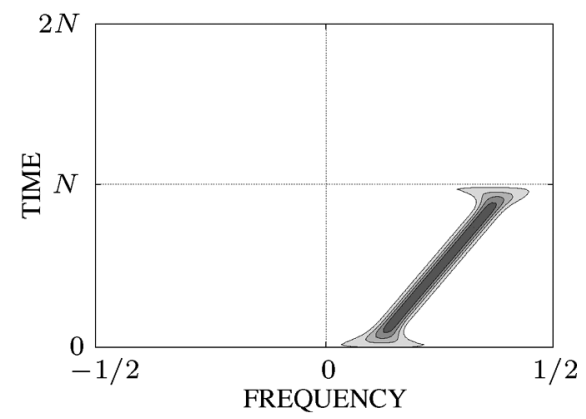

(b)

Fig. 3. Comparison of (a) AF-GDTFD and (b) proposed DTFD definition of the analytic LFM signal $z_{1}(n)$. Left plots are lag-independent kernel DTFDs, middle plots are Doppler-independent kernel DTFDs, and right plots are separable-kernel DTFDs. For the AF-GDTFD, any energy outside the bottom right quadrant is caused by aliasing.

$0 \leq f \leq 1 / 2$ because of the analytic signal's zero regions in (3) and (4). As the plots show, this is not so for the lag- and Doppler-independent kernel DTFDs; thus these DTFDs are aliased. For the separable-kernel DTFD, however, the kernel suppresses the aliasing energy. Thus, depending on the kernel structure, the AF-GDTFD may have little or even no aliasing energy. For example, we know that the Rihaczek distribution will be alias free. The proposed definition, however, remains alias free for all kernel types.

Note that because the AF-GDTFD is a full-plane distribution, the signal's energy is spread over the entire full plane. If we were to narrow the AF-GDTFD to the quarter-plane, then we would lose signal information and this quarter-plane AF-GDTFD would not satisfy many properties, such as the time or frequency marginals.

\section{CONCLUSION}

The proposed DTFD definition satisfies all desirable properties and is (approximately) equal to samples of the continuous WVD convolved with a time-frequency kernel. Neither the GDTFD nor the AF-GDTFD satisfies all desirable properties, and only the GDTFD approximates samples of the continuous WVD convolved with a kernel. Also, the proposed definition - unlike either the GDTFD or AF-GDTFD - contains a DWVD definition that satisfies important DWVD properties such as Moyal's formula and signal recovery. The proposed definition can be computed by simple and efficient algorithms. Matlab and Octave code to accompany this paper is available to download at http://www.ieeexplore.ieee.org/.

\section{APPENDIX}

\section{PROOFS FOR DTFD PROPERTIES}

We outline proofs for the set of DTFD properties from Section III-A. The complete proofs are in [18].
The proposed DTFD, in terms of the time-lag function $K^{\mathrm{C}}$ and kernel $G^{\mathrm{C}}$, is

$\rho^{\mathrm{C}}\left(\frac{n}{2}, \frac{k}{2 N}\right)=\frac{1}{2 N} \sum_{m=0}^{2 N-1} \sum_{p=0}^{2 N-1} K^{\mathrm{C}}\left(\frac{p}{2}, m\right) G^{\mathrm{C}}\left(\frac{n-p}{2}, m\right) \mathrm{e}^{-\mathrm{j} \pi m k / N}$

and, in terms of the Doppler-frequency function $\mathcal{K}^{\mathrm{C}}$ and kernel $\mathcal{G}^{\mathrm{C}}$, is $\rho^{\mathrm{C}}\left(\frac{n}{2}, \frac{k}{2 N}\right)=\frac{1}{4 N^{2}} \sum_{l=0}^{2 N-1} \sum_{q=0}^{2 N-1} \mathcal{K}^{\mathrm{C}}\left(\frac{l}{N}, \frac{q}{2 N}\right) \mathcal{G}^{\mathrm{C}}\left(\frac{l}{N}, \frac{k-q}{2 N}\right) \mathrm{e}^{\mathrm{j} \pi l n / N}$

for $n, k=0,1, \ldots, 2 N-1$, where $\mathcal{K}^{\mathrm{C}}(l / N, k / 2 N)=Z((k+$ $l) / 2 N) \bar{Z}((k-l) / 2 N)$. The $2 N \times 2 N$ DTFD in (14) and (15) is a periodic extension, using (11), of the $2 N \times N$ DTFD in (10).

P1) Nonnegativity: Because $h(n / 2)$ and $G^{\mathrm{C}}(n / 2, m)$ are zero when $n / 2$ is not an integer, the kernel is nonzero for $G^{\mathrm{C}}(n, 2 m)$ only. Therefore, using (14), the DTFD is zero when $n / 2$ is not an integer; that is, $\rho^{\mathrm{C}}(n+1 / 2, k / 2 N)=0$. When $n / 2$ is an integer and as $G^{\mathrm{C}}(n, 2 m)=h(n+m) \bar{h}(n-m)$

$$
\begin{array}{r}
\rho^{\mathrm{C}}\left(n, \frac{k}{2 N}\right)=\frac{1}{2 N} \sum_{p=0}^{N-1} \sum_{m=0}^{N-1} z(n-p+m) \bar{z}(n-p-m) \\
\cdot h(p+m) \bar{h}(p-m) \mathrm{e}^{-\mathrm{j} 2 \pi m k / N} .
\end{array}
$$

After some manipulation

$$
\begin{aligned}
\rho^{\mathrm{C}}\left(n, \frac{k}{2 N}\right) & =\frac{1}{2 N}\left|\sum_{a=0}^{N-1} z(2 a) \bar{h}(n-2 a) \mathrm{e}^{-\mathrm{j} 2 \pi a k / N}\right|^{2} \\
& +\frac{1}{2 N}\left|\sum_{a=0}^{N-1} z(2 a+1) \bar{h}(n-2 a-1) \mathrm{e}^{-\mathrm{j} 2 \pi a k / N}\right|^{2}
\end{aligned}
$$

and the DTFD is therefore nonnegative. 
P2) Time marginal: Simply applying the property's kernel constraint to (14) results in

$$
\sum_{k=0}^{2 N-1} \rho^{\mathrm{C}}\left(n, \frac{k}{2 N}\right)=K^{\mathrm{C}}(n, 0)=|z(n)|^{2} .
$$

P3) Frequency marginal: Applying the property's kernel constraint to (15) results in

$$
2 N \sum_{n=0}^{2 N-1} \rho^{\mathrm{C}}\left(\frac{n}{2}, \frac{k}{2 N}\right)=\mathcal{K}^{\mathrm{C}}\left(\frac{0}{N}, \frac{k}{2 N}\right)=\left|Z\left(\frac{k}{2 N}\right)\right|^{2} .
$$

P4) Time support: The DTFD equals the DFT of the smoothed time-lag function $R^{\mathrm{C}}(n / 2, m)$, where

$$
R^{\mathrm{C}}\left(\frac{n}{2}, m\right)=\rho^{\mathrm{C}}\left(\frac{n}{2}, m\right) \underset{n}{\circledast} G^{\mathrm{C}}\left(\frac{n}{2}, m\right) .
$$

To satisfy time support, $R^{\mathrm{C}}$ must have the same time support as $K^{\mathrm{C}}$; that is, if $K^{\mathrm{C}}(n / 2, m)=0$ for $n<2 n_{1}$ and $n>2 n_{2}$, then the property requires that $R^{\mathrm{C}}(n / 2, m)=0$ for $n<2 n_{1}$ and $n>2 n_{2}$. The property's kernel constraint ensures that $R^{\mathrm{C}}(n / 2, m)$ has the same time support as $K^{\mathrm{C}}(n / 2, m)$, and thus the DTFD satisfies the property.

P5) Frequency support: The same process as for the previous property but swapping the time-lag domain for the Doppler-frequency domain.

P6) Instantaneous frequency: Applying the property's kernel constraint to (14)

$\sum_{k=0}^{2 N-1} \rho^{\mathrm{C}}\left(n, \frac{k}{2 N}\right) \mathrm{e}^{\mathrm{j} 2 \pi k / N}=a K^{\mathrm{C}}(n, 2)=a z(n+1) \bar{z}(n-1)$.

Using the polar notation $z(n)=A(n) \exp [\mathrm{j} \varphi(n)]$

$\arg \left[\sum_{k=0}^{2 N-1} \rho^{\mathrm{C}}\left(n, \frac{k}{2 N}\right) \mathrm{e}^{\mathrm{j} 2 \pi k / N}\right]=\varphi(n+1)-\varphi(n-1)$

which forms the basis for the proof [19].

P7) Group delay: Applying the property's kernel constraint to (15)

$$
\sum_{n=0}^{2 N-1} \rho^{\mathrm{C}}\left(\frac{n}{2}, \frac{k}{2 N}\right) \mathrm{e}^{-\mathrm{j} \pi n / N}=a \mathcal{K}^{\mathrm{C}}\left(\frac{1}{N}, \frac{k}{2 N}\right)
$$

and, using the polar notation $Z(k / 2 N)=b(k) \exp [\mathrm{j} \theta(k)]$

$\arg \left[\sum_{n=0}^{2 N-1} \rho^{\mathrm{C}}\left(\frac{n}{2}, \frac{k}{2 N}\right) \mathrm{e}^{-\mathrm{j} \pi n / N}\right]=\theta(k+1)-\theta(k-1)$

which forms the basis of the proof.

P8) Moyal's formula: To prove this property, we use the identity

$$
\begin{aligned}
& \sum_{n=0}^{2 N-1} \sum_{k=0}^{2 N-1} \rho_{x}^{\mathrm{C}}\left(\frac{n}{2}, \frac{k}{2 N}\right) \bar{\rho}_{y}^{\mathrm{C}}\left(\frac{n}{2}, \frac{k}{2 N}\right) \\
&=\sum_{l=0}^{2 N-1} \sum_{m=0}^{2 N-1} S_{x}\left(\frac{l}{N}, m\right) \bar{S}_{y}\left(\frac{l}{N}, m\right)
\end{aligned}
$$

where $S(l / N, m)$ is the smoothed discrete ambiguity function, the two-dimensional DFT of $\rho^{\mathrm{C}}(n / 2, k / 2 N)$. Expanding
$S(l / N, m)$ in terms of time-lag functions and applying the property's kernel constraint, (16) is equal to

$$
\begin{aligned}
\frac{1}{2 N} \sum_{a=0}^{2 N-1} \bar{x}(a) y(a) \sum_{m=0}^{2 N-1} x(a+m) & \bar{y}(a+m) \\
= & \frac{1}{2 N}\left|\sum_{a=0}^{2 N-1} x(a) \bar{y}(a)\right|^{2}
\end{aligned}
$$

thus proving the property.

P9) Signal recovery: The DTFD collapses to the DWVD after applying the property's kernel constraint to (14). The proof for the DWVD is in [8].

\section{REFERENCES}

[1] T. Claasen and W. Mecklenbräuker, "The Wigner distribution-A tool for time-frequency signal analysis, Part II: Discrete-time signals," Philips J. Res., vol. 35, pp. 276-350, 1980.

[2] D. Chan, "A non-aliased discrete-time Wigner distribution for timefrequency signal analysis," in Proc. IEEE Int. Conf. Acoust., Speech, Signal Process. (ICASSP-82), May 1982, vol. 7, pp. 1333-1336.

[3] F. Peyrin and R. Prost, "A unified definition for the discrete-time, discrete-frequency, and discrete-time/frequency Wigner distributions," IEEE Trans. Acoust., Speech, Signal Process., vol. ASSP-34, pp. 858-866, Aug. 1986.

[4] A. H. Nuttall, "Alias-free Wigner distribution function and complex ambiguity function for discrete-time samples," Naval Underwater Systems Center (NUSC), New London, CT, Tech. Rep. 8553, Apr. 1989.

[5] B. Boashash and A. Reilly, "Algorithms for time-frequency signal analysis," in Time-Frequency Signal Analysis: Methods and Applications, B. Boashash, Ed. Melbourne 3205, Australia: Wiley, 1992, ch. 7, pp. $163-181$

[6] J. Jeong and W. Williams, "Alias-free generalized discrete-time time-frequency distributions," IEEE Trans. Signal Process., vol. 40, pp. 2757-2765, Nov. 1992.

[7] J. C. O'Neill, P. Flandrin, and W. J. Williams, "On the existence of discrete Wigner distributions," IEEE Signal Process. Lett., vol. 6, no. 12, pp. 304-306, Dec. 1999.

[8] J. O'Toole, M. Mesbah, and B. Boashash, "A discrete time and frequency Wigner-Ville distribution: Properties and implementation," in Proc. Int. Conf. Digital Signal Process. Commun. Syst., Noosa Heads, Australia, Dec. 19-21, 2005 [Online]. Available: http://www.eprints.qut.edu.au/2607/

[9] J. C. O'Neill and W. J. Williams, "Shift covariant time-frequency distributions of discrete signals," IEEE Trans. Signal Process., vol. 47, pp. 133-146, Dec. 1999.

[10] A. H. Costa and G. F. Boudreaux-Bartels, "An overview of aliasing errors in discrete-time formulations of time-frequency representations," IEEE Trans. Signal Process., vol. 47, pp. 1463-1474, May 1999.

[11] J. M. O'Toole, M. Mesbah, and B. Boashash, "A new discrete analytic signal for reducing aliasing in the discrete Wigner-Ville distribution," IEEE Trans. Signal Process., vol. 56, pp. 5427-5434, Nov. 2008.

[12] B. Boashash, "Part I: Introduction to the concepts of TFSAP," in TimeFrequency Signal Analysis and Processing: A Comprehensive Reference, B. Boashash, Ed. Oxford, U.K.: Elsevier, 2003, ch. 1-3, pp. $3-76$.

[13] A. V. Oppenheim and R. W. Schafer, Discrete-Time Signal Processing. Englewood Cliffs, NJ: Prentice-Hall, 1999, 07458.

[14] Time-Frequency Signal Analysis and Processing: A Comprehensive Reference, B. Boashash, Ed. Oxford, U.K.: Elsevier, 2003.

[15] D. Slepian, "On bandwidth," Proc. IEEE, vol. 64, no. 3, pp. 292-300, 1976.

[16] J. M. O'Toole, M. Mesbah, and B. Boashash, "A new definition of discrete quadratic time-frequency distributions," in Proc. 16th Eur. Signal Process. Conf. (EUSIPCO-08), Lausanne, Switzerland, Aug. 25-29, 2008.

[17] J. M. O'Toole, M. Mesbah, and B. Boashash, "Efficient algorithms for discrete time-frequency distributions," in Proc. Appl. Comput. Conf. (ACC'08), Istanbul, Turkey, May 27-30, 2008, pp. 310-315.

[18] J. M. O'Toole, M. Mesbah, and B. Boashash, "Proofs for discrete timefrequency distribution properties," UQ Centre for Clinical Research and Perinatal Research Centre, University of Queensland, Australia, Technical Report UQCCR-2009-06-09, Jun. 2009 [Online]. Available: http://espace.library.uq.edu.au/view/UQ:178641

[19] B. Boashash, "Time-frequency signal analysis," in Advances in Spectrum Estimation, S. Haykin, Ed. Englewood Cliffs, NJ: Prentice-Hall, 1991, ch. 9, pp. 418-517. 